\title{
Una Summer school in pandemia. Introduzione al dossier
}

di Agnese Portincasa and Aldo Gianluigi Salassa

Dic 2, 2020 | Emergenza e (nuova) normalità, In evidenza $|\underline{0}|$

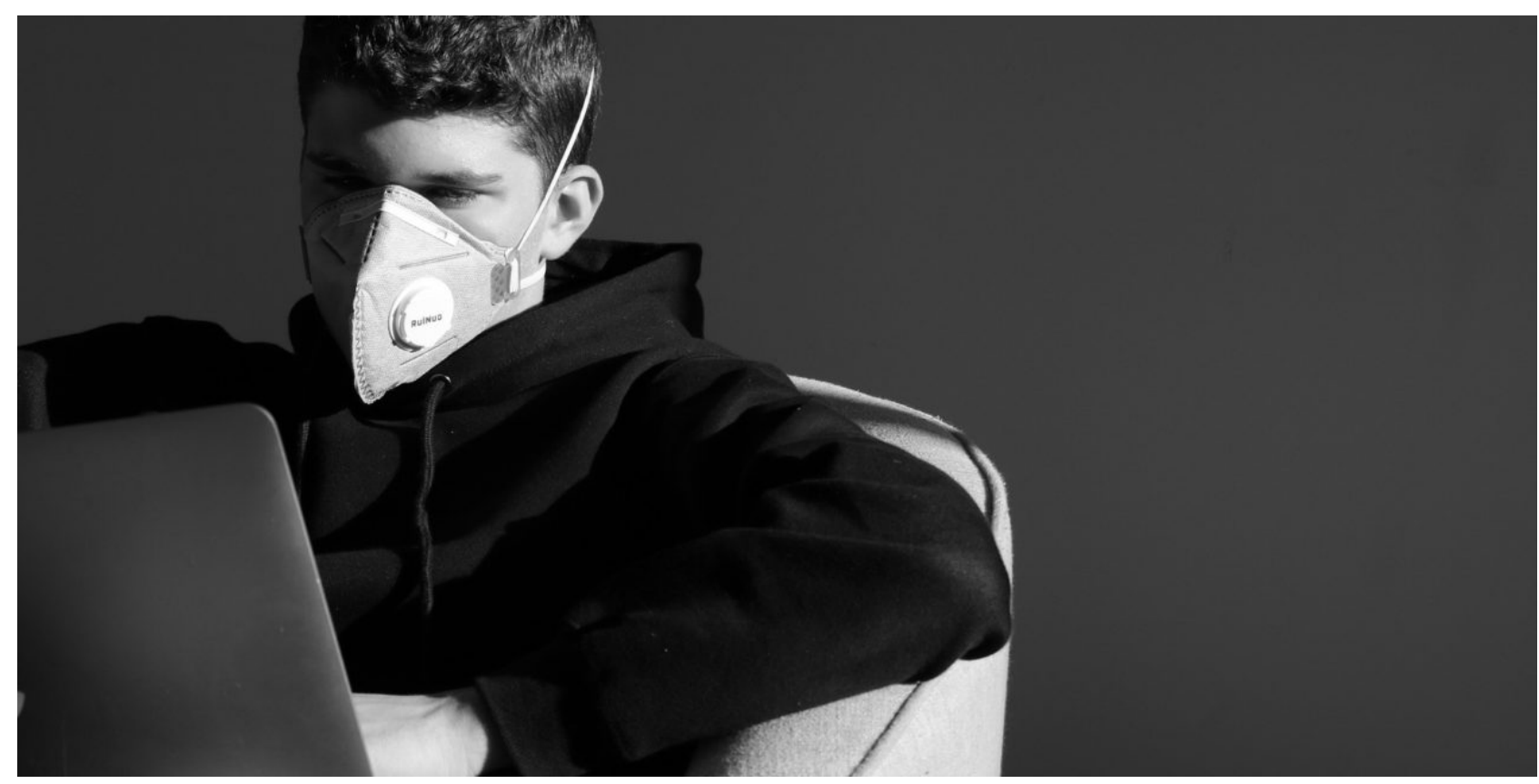

dida

\begin{abstract}
L'edizione 2020 della Summer School dell'Istituto Nazionale Ferruccio Parri e della rete di istituti della Resistenza ha avuto come titolo Emergenze e nuove normalità, con un sottotitolo che precisava il percorso proposto: Didattica della storia e educazione alla cittadinanza in tempi di virus. Si è trattato di un'edizione per così dire speciale, perché svolta interamente a distanza a causa delle limitazioni imposte dalla pandemia in corso.
\end{abstract}

Una premessa indispensabile

Nel febbraio 2020 avevamo già un tema (l'educazione civica a scuola), una sede (Torino) e delle date (l'ultimo fine settimana di agosto) per la nostra Summer School, giunta alla sua settima edizione. Poi l'emergenza Covid-19 ha rimesso tutto in forse e per molti giorni la tentazione di saltare l'appuntamento in attesa di tempi migliori, è stata molto forte. Molti enti formatori hanno scelto questa strada, in un certo senso comprensibilmente.

Le questioni sul tavolo erano tante: come lavorare a distanza con dei docenti senza averlo mai fatto prima? Come gestire la distanza salvando la laboratorialità che è il nostro elemento di riconoscibilità? Avremmo saputo gestire gli aspetti tecnici di un evento di formazione da remoto?

Nonostante le difficoltà del momento nella primavera e in pieno lockdown prevalse il desiderio di non abbandonare i docenti in una fase così critica, in un momento in cui la formazione intesa come 
incontro, anche se su piattaforma, avrebbe potuto significare ricominciare a confrontarsi dopo mesi di segregazione domestica, affrontare questioni di metodo, iniziare a ragionare di Didattica a Distanza o di Didattica Digitale Integrata.

L'emergenza Covid-19 sull'evento Summer School non ha comportato soltanto la scelta di un preciso format e di una specifica articolazione temporale (dilatata su più settimane), ma ha imposto anche, per così dire, una determinata agenda: il comitato scientifico e organizzatore non ha potuto né voluto - fare a meno di tenere conto, nel definirne gli argomenti e gli snodi concettuali, dei temi posti all'ordine giorno dal dibattito pubblico attivato dal virus; cercando peraltro di declinarli ed elaborali in una dimensione formativa, in vista di una loro possibile traduzione in percorsi didattici.

Alle tre interviste pre-Summer (a Gustavo Zagrebelsky, Luciano Floridi e Pier Cesare Rivoltella: anche questa una novità) è toccato il compito di aprire la riflessione di più ampio respiro: un costituzionalista, un filosofo dell'informazione e un esperto di didattica digitale ci hanno condotto a analizzare l'emergenza nelle sue molteplici sfaccettature.

La Summer School vera e propria - da fine agosto a fine settembre - si è caratterizzata per l'alternarsi di due momenti, replicati su cinque settimane: ogni giovedì pomeriggio un approfondimento affidato ad esperti seguito, il pomeriggio successivo, da una formazione attiva, che ha coinvolto alcuni Istituti della rete chiamati a presentare lavori di ricerca didattica già elaborati, congruenti con i temi affrontati e da approfondire in attività di laboratorio con gli iscritti. Tutti: esperti, docenti e tutor, sono stati chiamati a sviluppare le loro riflessioni e gli spunti laboratoriali da alcune parole chiave presentate in coppia ed "estratte" dal dibattito svoltosi attorno alla pandemia.

Così pensata e congegnata, la scuola ha riscosso, non solo dal punto di vista numerico, un notevole successo; a riprova dell'attenzione di cui gli Istituti di tutta Italia godono presso il pubblico dei docenti in servizio a scuola. La Summer, inoltre, ha inequivocabilmente enfatizzato la ricchezza del lavoro di rete che è presente nell'alveo dell'Istituto Nazionale: l'utilizzazione del patrimonio di esperienze accumulato dagli Istituti nei territori è stato rielaborato e riadattato secondo le esigenze di un setting online che ha inevitabilmente condizionato le modalità della comunicazione attraverso una piattaforma digitale. Esperienza professionale e relazionale cui tutto il personale della rete ha potuto accedere, azzerando così ogni confine fra centri e periferie.

La sfida della Summer ha avuto anche il merito di dare sempre maggior forza all'idea - nata e maturata in tutte le edizioni precedenti - di fare formazione docenti nel flusso del presente-passatopresente. In tempi di pandemia tale modalità ha finito per diventare addirittura urgente. Come molto puntualmente ha rilevato Salvatore Adorno nella tavola rotonda iniziale: chi si occupa di storia sa bene che le domande nascono dal presente, e questo presente è talmente ingombrante che è impossibile sfuggirgli.

La settima Summer School a distanza è stata una sfida. Ma ci sembra si possa dire che sia stata una sfida vinta. I docenti iscritti erano sempre presenti, nessuna riduzione numerica nel corso di cinque settimane, ma soprattutto abbiamo avuto un ottimo riscontro per i workshop a distanza. Il lavoro a piccoli gruppi nelle breakout rooms ha funzionato meglio di quanto noi stessi potessimo credere e lo scambio con i docenti è stato proficuo.

Certo, la presenza è mancata, ma questa è ormai un'osservazione che non aggiunge nulla. La presenza manca a tutti e in ogni contesto della nostra quotidianità, e proprio questo è un portato dell'evento pandemico. Misurarci con gli scenari aperti dal nostro presente sul nostro futuro è doveroso. 


\section{Che cosa abbiamo imparato? Per una cassetta degli attrezzi}

In questo contributo di apertura del dossier, chi scrive vuole provare a toccare quello che, in ogni singola relazione esperta, potrebbe essere portato sulla scrivania del docente come suggestione.

In prima battuta e in termini generali abbiamo trovato conferma di quanto indispensabile sia un approccio interdisciplinare quando ci si trova di fronte - nella formazione come nella didattica - a fenomeni complessi sotto il profilo storico e sociale quali sono appunto le pandemie, le catastrofi "naturali", le guerre, le grandi crisi economiche globali, con le inevitabili "rotture" che esse determinano nella normalità della vita quotidiana. Per questo motivo nella Summer abbiamo voluto mescolare interventi di carattere più marcatamente storico come quelli di Patrizia Gabrielli, Matteo Pasetti, Giovanni De Luna, Emanuele Felice, Giovanni Gozzini con altri sguardi, come quelli di Laura Boella, Anna Loretoni, Lea Ipy, Enrico Manera. Si è trattato di un intreccio che non soltanto ha messo in evidenza la complessità dei problemi affrontati, ma ha stimolato pensieri divergenti rispetto a quelli che si muovono negli ambiti puramente disciplinari e della metodologia didattica dedicata.

Nelle nostre Summer, anche in passato, si è parlato spesso di una cassetta degli attrezzi: un'immagine che richiama un gesto pratico di utilizzo di saperi e metodologie spendibili nella quotidianità didattica; un armamentario, insomma, di idee come oggetti utili/strumenti per innescare virtuosi tracciati per gli apprendimenti.

Nel dossier pubblicheremo la rielaborazione, a cura dei docenti universitari coinvolti negli appuntamenti del giovedì, delle lezioni svolte.

Ma vogliamo concludere questa introduzione al dossier provando a individuare alcuni nodi dei pensieri stimolati dalle relazioni e offrirli come strumenti che ognuno potrà mettere nella propria cassetta di docente professionista.

Seguiremo il filo dello svolgimento temporale delle cinque settimane della Summer.

\section{PRIMA SETTIMANA - EMERGENZA/RICOSTRUZIONE}

Dalla relazione di Patrizia Gabrielli portiamo con noi l'idea di considerare il Covid-19 un evento periodizzante a tutti gli effetti e che stiamo vivendo in diretta, con la consapevolezza che lo sia. La pandemia si pone come momento nel quale il prima e il dopo si distinguono con una chiarezza difficilmente paragonabile ad altri eventi storici recenti. E' una brusca sterzata, dice Gabrielli.

Il suo sguardo ci ha molto intrigato: ha portato avanti tutta la sua relazione con lo sforzo notevole di leggere gli eventi del presente da storica, pur sapendo di non poterlo ancora fare. La sua idea, ad esempio, di raccogliere e utilizzare le memorie del presente - sia le fonti orali, sia le scritture autonarrative (le autobiografie, le memorie, le lettere, i diari) per la costruzione della storia futura ci pare ottima e offre molti spunti per la didattica. Ciascuno, nella sua classe, può raccogliere questa tipologia di memorie, costruire un diario della crisi.

Dalla relazione di Matteo Pasetti ci resta il j'accuse nel mostrarci come la storiografia e la manualistica si occupino pochissimo delle crisi che somigliano a quella che stiamo vivendo. Nei 
nostri libri di storia la conta dei morti è sempre un argomento di rilievo, ma fra questi non ci sono quasi mai i morti delle pandemie, dei terremoti, delle catastrofi (si pensi solo a Chernobyl). Pasetti ci ha inoltre parlato dell'ambigua commistione fra la memoria fredda generata da questi eventi e la spettacolarizzazione della morte che riguarda la fase dell'emergenza. I morti delle emergenze sono interessanti fino a che bucano il video dei media - e anzi la spettacolarizzazione della morte è una delle conseguenze della rivoluzione mediatica propria del Novecento - ma poi scompaiono, o quasi.

\section{SECONDA SETTIMANA - LIBERTÀ/RESPONSABILITÀ}

Della relazione di Laura Boella ci ha colpito la sua proposta di rovesciare il rapporto fra libertà e responsabilità - tema caldissimo che, non a caso, è stato pochi giorni dopo al centro del botta e risposta fra il premier britannico Boris Johnson e il presidente Sergio Mattarella. In classe, un percorso molto proficuo di educazione civica potrebbe essere proprio improntato su quanto proposto dalla filosofa morale: una nuova idea di libertà, a fondamento della quale c'è la responsabilità. Una libertà responsabile capace di salvaguardare l'importanza e il valore dell'individuo contro la logica della massa e i processi spersonalizzanti delle odierne eco-chambers tipiche della comunicazione social. Occorre, sostiene Boella, interrompere i flussi che passano sopra le nostre teste, le derive, i processi automatici; occorre scompaginare la carte, rifiutare gli anestetici che ci vengono propinati in dosi massicce e che ci fanno dimenticare la realtà. Tutto questo senza accampare nessun eroismo e nessuna retorica. Essere presenti e liberi nel proprio tempo può assumere le forme più diverse: lo studio, la ricerca, mettere al mondo un figlio, fare bene una lezione, vedere le parole oltre l'opacità comunicativa.

Della relazione di Giovanni De Luna portiamo con noi moltissime cose, in particolare il modo in cui ci ha parlato della Resistenza, quando, a proposito della parola chiave "responsabilità", ha definito la generazione di quelli che l'hanno fatta come degli irresponsabili marci. Gente che aveva voluto e saputo disobbedire a tutte le regole.

E tuttavia questa soggettività continuamente dispiegata non è, per De Luna, solo quel "diavolo in corpo" necessario per scuotere una società da vent'anni di obbedienza e di conformismo, ma si trasforma e si concretizza nel miracolo della Costituzione.

Per quella generazione l'idea della libertà non si è limitata a togliere di mezzo il vecchio, ma ha saputo costruire il nuovo fissandolo nelle regole della propria idea di libertà. Interessante, e un poco amara, la riflessione finale dello storico: la sua generazione - quella del Sessantotto - ha conosciuto anch'essa la "pulsione irrefrenabile alla rottura delle regole e alla disobbedienza", ma ha pagato un prezzo altissimo proprio all'incapacità di costruire, sulla distruzione delle vecchie regole, regole nuove.

\section{TERZA SETTIMANA - CENTRO/PERIFERIA}

Della relazione di Anna Loretoni ci ha colpito la sua riflessione sul vantaggio epistemico che può essere dato da una condizione di marginalità. I soggetti periferici come soggetti eccentrici sono portatori di una visione critica di valore decostruttivo. Il margine (come nel pensiero di Bell Hooks) non è solo luogo di privazione e di mancanza, un luogo di assenza di potere, ma è anche un luogo da cui si può vedere qualcosa che chi sta al centro della mappa concettuale non vede. È un esercizio che potrebbe essere portato a scuola in percorsi di educazione di genere (ma non solo) capaci di 
uscire dalle narrazioni mainstream della marginalità, che spesso ci orientano a ragionare esclusivamente di violenza, di bias e di stereotipizzazione.

Della relazione di Giovanni Gozzini ci resta la suggestione di provare a spiegare quella difficilissima cosa che è la globalizzazione, semplificandola: restringendo il concetto e collocandolo nel tempo. Il tempo sarebbe quello degli ultimi due secoli che vedono alternarsi fasi di globalizzazione e de-globalizzazione. Per restringere il concetto, invece, può essere utile partire da esempi. Splendido e misconosciuto quello della vita di Elizabeth Jane Cochran, giornalista americana che nel 1899 - quasi vent'anni dopo la pubblicazione de Il giro del mondo in ottanta giorni di Jules Verne - il giro del mondo lo fa davvero, da donna e in soli settantatré giorni. Una storia didatticamente piena di spunti anche per parlare arrivare a parlare di storia locale (a Torino, ad esempio, Cochran fu accolta da un manipolo di pie donne che tentarono di assaltare il treno su cui viaggiava).

\section{QUARTA SETTIMANA - STATO/MERCATO}

Della relazione di Emanuele Felice ci rimane una visione dei rapporti intercorsi tra stato e mercato in Occidente meno schematica e prevedibile di quella che siamo soliti trovare nei manuali. Tra lo "stato minimo" d'impronta liberale di fine Ottocento e lo stato succube del mercato degli ultimi decenni, c'è di mezzo una lunga fase - che va dal periodo infrabellico agli anni Settanta - in cui si afferma il modello di successo del welfare state, successivamente sottoposto a un (parziale) smantellamento. Oggi la pandemia sta rimescolando le carte: accentua la crisi della cornice neoliberale che, in verità, era iniziata già prima dell'emergenza sanitaria, quando le forze del mercato non si erano dimostrate in grado di affrontare le conseguenze della transizione tecnologica, della crisi ambientale e climatica, della crescita delle disuguaglianze.

Interessante, inoltre, l'analisi cruda sulla difficile lettura, da parte della politica e della società italiane, dei nostri tempi. Non a caso - sostiene Felice - siamo il paese avanzato che spende meno in istruzione rispetto a PIL. Sul nostro declino pesano moltissimo le tre diseguaglianze che abbiamo più di altri nell'Eurozona: di generazione, di genere, di zona e di territori fra Nord e Sud.

Della relazione di Lea Ipy portiamo con noi il disagio che qualunque europeo nato prima degli anni Ottanta può provare nel sentire con tanta chiarezza come la retorica progressista in tutto il mondo continui a celebrare l'ideale di una cittadinanza che non esiste più, mentre non si parla mai della trasformazione che l'ha resa inefficace.

Nell'età di una cittadinanza restrittiva usata come merce, il politico non passa più dai canali standard della rappresentanza. È questa un'indicazione che potrebbe essere utile per decostruire l'idea stessa dei percorsi di educazione civica: non ci si può più permettere di presentare a studenti $\mathrm{e}$ studentesse un percorso concluso, guidato dall'idea che stiamo inesorabilmente marciando verso il migliore dei mondi possibili. Dopo Ipy ci viene da pensare che si potrebbe alzare l'asticella, focalizzando l'attenzione su cosa intendere oggi per rappresentanza politica e cittadinanza.

\section{QUINTA SETTIMANA - UGUAGLIANZA/DIFFERENZE}

Della relazione di Enrico Manera, dedicata a ricostruire le principali manifestazioni di discriminazione e de-umanizzazione che si sono verificate nella storia europea, portiamo con noi la 
cautela con cui maneggiare la parola identità, inflazionata oggi nel dibattito pubblico. L'identità, spiega Manera, è una finzione, un costrutto artificiale basato su un sentimento di appartenenza socialmente costruito; è un progetto politico, che alcune élite perseguono per la conquista del potere.

In tempo di negazionismi imperanti, sarebbe utile maturare la consapevolezza che la cultura europea è legata al razzismo e non è un caso dunque che oggi, nelle destre radicali, l'idea di razza continui a nascondersi dietro a termini presentabili come "etnicità" e "cultura". L'avversione nei confronti del diverso da Sé è servita in salsa bio-storica o etnico-culturalista o identitaria, che dir si voglia.

L'identità etnica e culturale, insomma, nient'altro sarebbero che postmoderne versioni dell'idea di razza, diffuse per preservare privilegi o diritti pensati di accesso limitato.

Anche in questo caso - come già per i temi affrontati da Ypi - sarebbe interessante ragionare su progetti di educazione civica che decostruiscano una certa mitologia progressista che tende a rimuovere le questioni con un ottimismo che si trasforma in miopia. 